\title{
VARIAÇÕES TÉRMICAS URBANAS NA CIDADE DE PRESIDENTE PRUDENTE
}

\author{
Gustavo Molina Momiy, Ivan Antunes Nobre Júnior, Luana Akinaga Marotti, Maria Alessandra \\ Bacaro Boscoli, Maria Eduarda Ramos
}

Universidade do Oeste Paulista - UNOESTE, curso de Arquitetura e Urbanismo, Presidente Prudente, SP. E-mail: lumarotti.vest@hotmail.com; allyboscoli@gmail.com

\section{RESUMO}

O presente trabalho analisou as variações de temperaturas durante cinco dias intercalados na cidade de Presidente Prudente do estado de São Paulo. Em um trajeto de 1,9 km, realizado na Rua Doze de Outubro, onde o primeiro ponto foi um posto de combustível e o último ponto foi o cruzamento com a Avenida Ademar de Barros. O objetivo deste estudo foi demonstrar as temperaturas dentro da mesma região da cidade e que a sua variação ocorre por diversos motivos. A metodologia utilizada se deu por medições nos pontos com um termômetro analógico acoplado em uma haste de madeira, esta que foi fixada na lateral de um veículo. Os resultados mostraram que diferentes usos na mesma região e a topografia contribuem com a variação da temperatura. Concluímos o quão singular é cada pequena região de uma cidade e por isso os estudos de conforto não podem ser tratados em grandes escalas.

Palavras-chave: desempenho, térmico, medições, variação, temperatura.

\section{URBAN THERMAL VARIATIONS IN THE CITY OF PRESIDENTE PRUDENTE}

\section{ABSTRACT}

The present work analyzed the temperature variations during five days interspersed in the city of Presidente Prudente of the state of São Paulo. On a route of $1.9 \mathrm{~km}$, carried out in the Street Doze de Outubro, where the first point was a fuel station and the last point was the intersection with Ademar de Barros Avenue. The objective of this study was to demonstrate the temperatures within the same region of the city and that its variation occurs for several reasons. The methodology used was by measurements at the points with an analogue thermometer coupled to a wooden rod, which was fixed to the side of a vehicle. The results showed that different uses in the same region and topography contribute to the temperature variation. We conclude how unique each small region of a city is and therefore the comfort studies can not be treated on a large scale.

\section{INTRODUÇÃO}

O estudo foi realizado na cidade de Presidente Prudente (fig. 1) do estado de São Paulo, na área central da cidade. A cidade possui aproximadamente 220 mil habitantes, com uma área de $560 \mathrm{~km}^{2}$ e possui um relevo que está inserido no Planalto Ocidental Paulista. Situada sobre um espigão divisor de águas e o sistema hidrográfico que drena o sítio urbano é constituído por pequenos cursos d'água. 
Fig.1 Imagem aérea de Presidente Prudente.

Fonte: Google Maps.

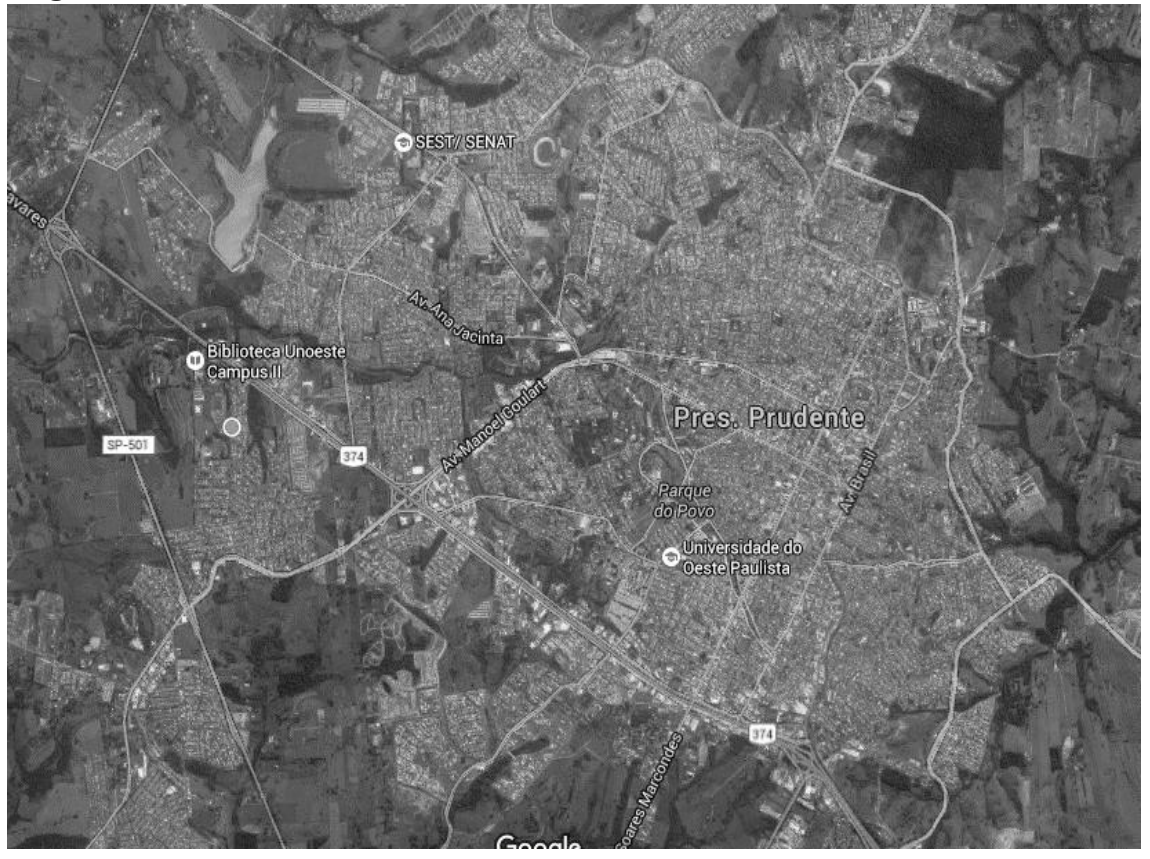

O transepto (fig. 2) tem uma extensão de um quilometro e novecentos metros, localizado na rua Doze de Outubro, onde o primeiro ponto foi um posto de combustível e o último ponto foi o cruzamento desta mesma rua com a avenida Ademar de Barros. A área tendo como uma marcante característica a alta densidade de edificações, com usos que se referem a comercial, serviços e residenciais em toda a sua extensão e por possuir pouca ou nenhuma vegetação arbórea em todo o trajeto estabelecido como objeto de estudo.

Fig. 2 Transepto em estudo.

Fonte: Google Maps.

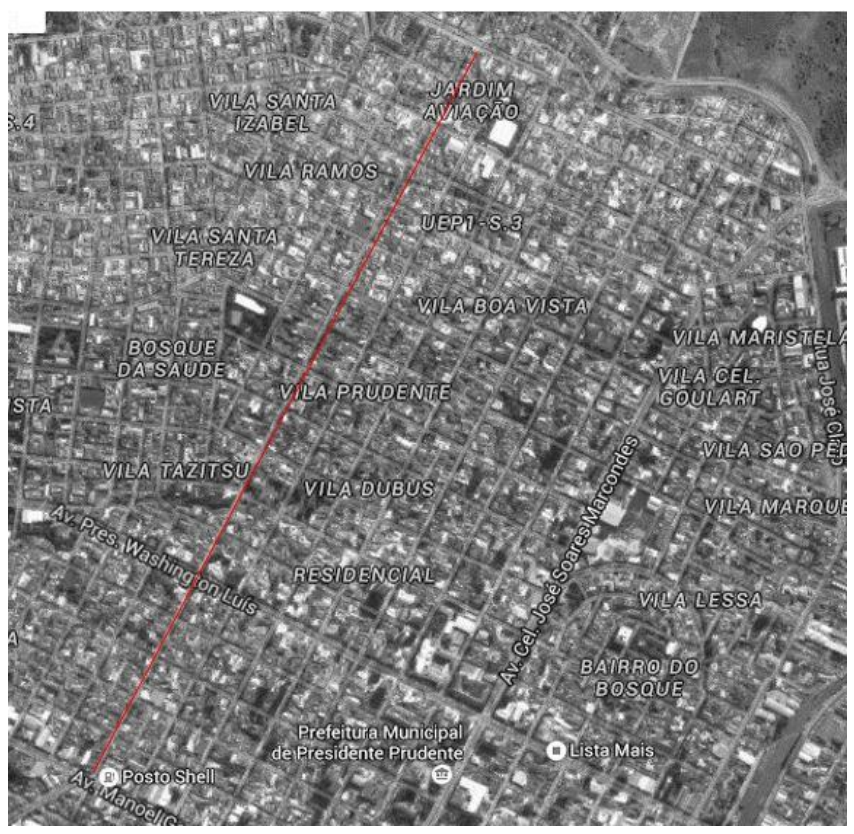

A movimentação de veículos e de pessoas é relativamente pequena nesta rua em estudo, em relação ao centro comercial da cidade, principalmente no horário adotado como o período de análise. Sua topografia se dá por uma declividade no meio do percurso, sendo este um fundo de vale. 
O clima de Presidente Prudente é uma consequência de um campo de alternância dos sistemas tropicais e polares, dominado por massas de ar tropical marítima. Porém a participação da Frente Polar Atlântica na gênese das chuvas é significativa, assim a cidade fica sujeita a frequentes invasões e perturbações frontais, mesmo quando as chuvas são mais frequentes, durante a primavera e o verão (AMORIM, 2005 apud. BARRIOS \& SANT'ANNA NETO, 1996).

A invasão das massas polares de trajetória continental e de ondas mais rigorosas de aquecimento pré-frontal provoca oscilação na temperatura. Enquanto a presença de sistemas estabilizadores de tempo no outono e no inverno provocam uma diminuição das chuvas (AMORIM, 2005 apud. BARRIOS \& SANT'ANNA NETO, 1996).

O objetivo deste estudo foi levantar questões pertinentes aos diferentes tipos de uso de e ocupação do solo, associados às características do relevo, que provocam mudanças na qualidade ambiental e mostrar o quão singular é cada região da mesma cidade, assim concluindo que se deve fazer estudos de conforto para cada caso, pois possuem diferenças mesmo em pequenas regiões da mesma cidade.

O estudo das temperaturas ainda permite a deteç̧ão de ilhas de calor, que ao saber onde estão é possível trabalhar com o planejamento urbano de maneira que ajude amenizar as temperaturas e diminuir este problema que é uma frequente nas cidades contemporâneas.

\section{METODOLOGIA}

No presente estudo das variações térmicas urbanas de Presidente Prudente a metodologia para a determinação das variáveis na temperatura consistiu na definição de um trajeto, com 18 pontos em toda a sua extensão, utilizando termômetros analógicos, sendo este fixo em uma haste de madeira com comprimento de 1,5 metro e acoplado na lateral de um veículo.

O veículo saiu do primeiro ponto, localizado no início da rua Doze de Outubro, em frente ao posto de combustível, passando pelo fundo de vale e chegando à sua extremidade, o seu cruzamento com a avenida Ademar de Barros. As medições foram efetuadas entre os dias dois de maio de 2016 ao dia 10 de maio de 2016, em dias alternados, sempre iniciando às 20 horas e tendo uma duração média de 45 minutos, horário escolhido por não apresentar grandes alterações na temperatura, pois o percurso possui um tempo de execução considerável.

A coleta das temperaturas se deu em cada um dos 18 pontos, sendo que em cada ponto o carro deveria permanecer parado durante dois minutos para adaptação da temperatura externa. O tempo gasto durante a medição não podia ultrapassar uma hora, metodologia que foi adaptada com base nos trabalhos de Oke e Maxwell (1975); Gomez e Garcia (1984); Johnson (1985); Pitton (1997).

Durante o período de medições houve a chegada de uma frente fria com chuvas na cidade, tendo assim influenciado diretamente as temperaturas analisadas. Para análise dos resultados foram feitas comparações entre as temperaturas dos mesmos pontos nos dias em que foram feitas as coletas dos dados.

\section{RESULTADOS}

É possível observar que nos cinco dias em que ocorreram as medições, a variação de temperatura não se manteve estável entre os pontos, assim como nos dias alternados. Tendo como justificativa a diferença topográfica entre os pontos e a quantidade de edificações e seus usos. A diferença de temperatura entre os dias em que foram feitas as medições se tem pelo fato de que ocorreu a chegada de uma frente fria na cidade, com chuvas. Tendo iniciado alguns dias antes das medições e com o passar dos cinco dias as temperaturas tiveram grandes variações.

Com essas variações da temperatura acaba ocorrendo o deslocamento do equilíbrio da reação endotérmica quando a temperatura aumenta e quando a temperatura diminui ocorre o deslocamento da reação exotérmica. 
Podemos observar os gráficos das variações climáticas entre os pontos em um mesmo dia de medições (cada gráfico representa um dia):

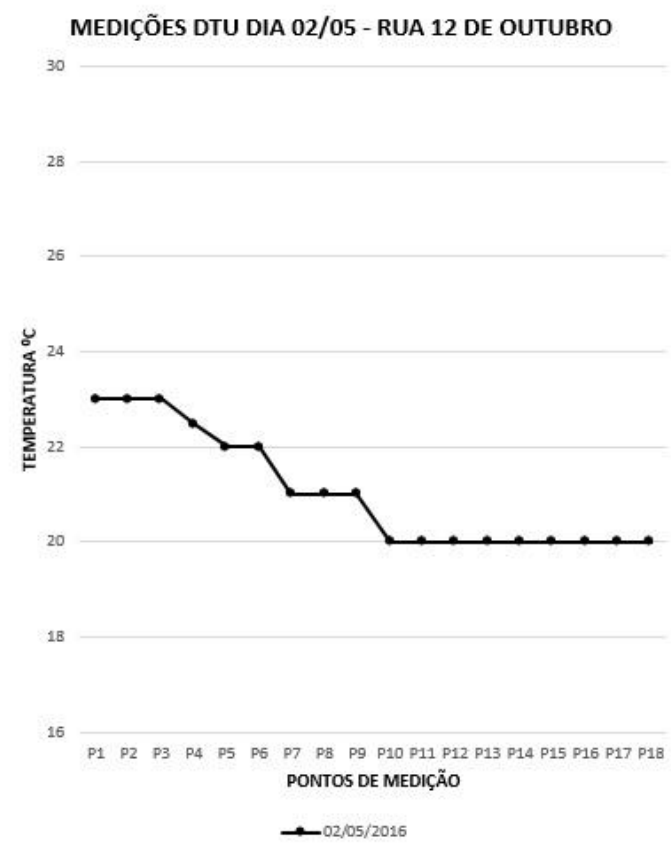

No primeiiro grafico pode-se verificar que a variação começa a ocorrer ente os pontos 3 e 10, sendo que antes e após esses pntos a temperatura se mateve estavel.

MEDIÇÕES DTU 04/05 - RUA 12 DE OUTUBRO

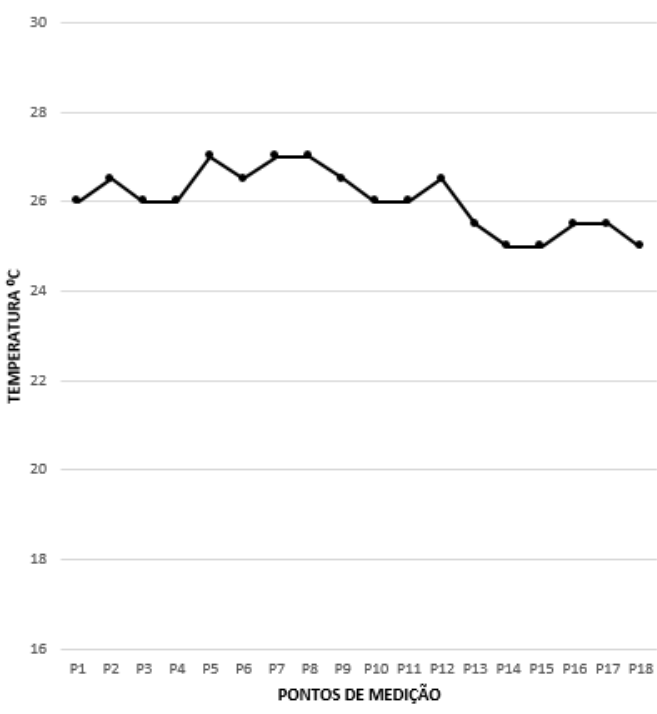

No segundo grafico pode-se verificar que a variação se mantem constante entre os pontos 1 e 18. 
MEDIÇÕES DTU 06/05 - RUA 12 DE OUTUBRO

30

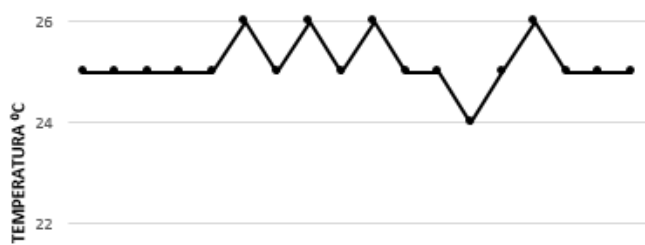

20

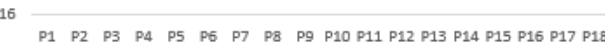
PONTOS DE MEDIÇÃO

-06/05/2016
No terceiro grafico pode-se verificar que a variação começa a ocorrer ente os pontos 5 e 16, sendo que antes e após esses pontos a temperatura se mateve estavel.
MEDIÇÕES DTU 08/05 - RUA 12 DE OUTUBRO$$
30
$$

28

26

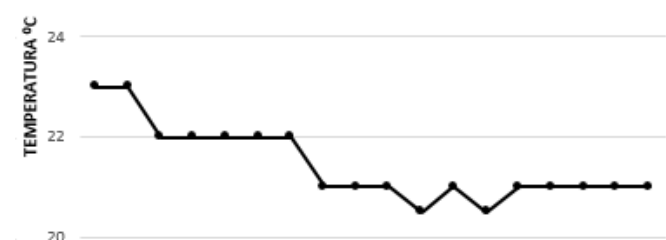

18

16

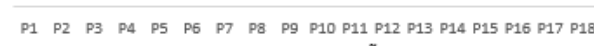
PONTOS DE MEDIÇÃO
No quarto grafico podes-se verificar que a variação começa a ocorrer ente os pontos 3 e 14, sendo que antes e após esses pontos a temperatura se mateve estavel. 
MEDIÇÕES DTU 10/05 - RUA 12 DE OUTUBRO

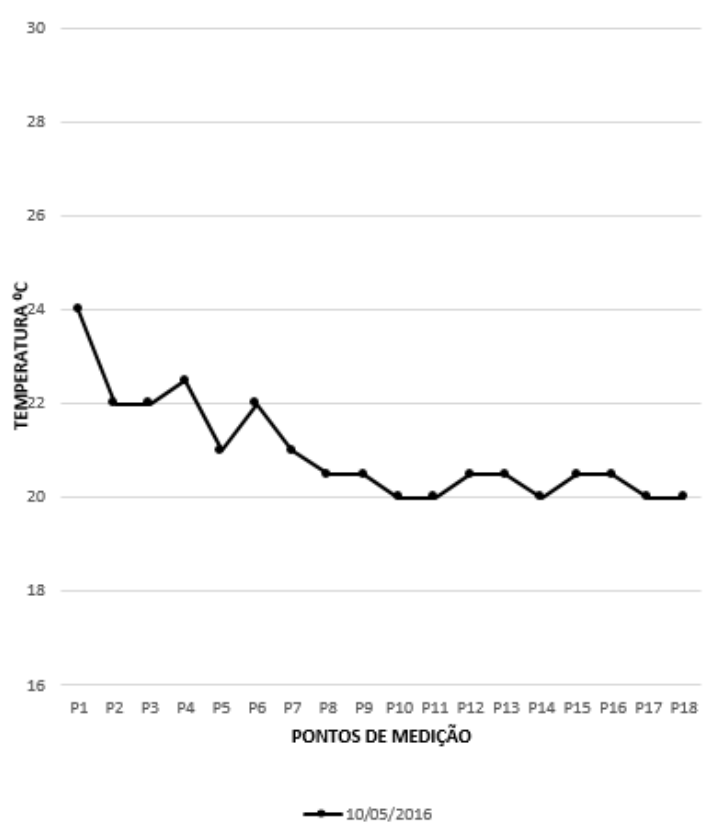

No quinto grafico podesse verificar que a variação se mantem constante entre os pontos 1 e 18 .

\section{DISCUSSÃO}

As coletas de dados foram realizadas no ano de 2016, em um período de 8 dias: de 2 a 10 de maio, intercaladamente. Escolheu-se 18 pontos, com espaçamento médio de 100 metros entre um e outro, para as coletas de temperatura. Os pontos se diferenciavam, principalmente, nos tipos de uso e topografia.

Foi possível observar que nos cinco dias em que ocorreram as medições, a variação de temperatura não se manteve estável entre os pontos. Notamos que a diferença topográfica entre e a quantidade de edificações e seus usos foram os principais fatores da variação.

Com o intuito de verificar melhor a variabilidade da temperatura do ar, foi estruturado um banco de dados em função dos dias de medição. Essa análise de agrupamento foi feita pelo grupo para entender melhor o microclima da rua.

\begin{tabular}{|c|c|c|c|c|c|c|c|c|c|}
\hline \multicolumn{10}{|c|}{ MEDIÇÕES DTU - RUA 12 DE OUTUBRO } \\
\hline \multicolumn{2}{|r|}{ DIA 02/05 } & \multicolumn{2}{|r|}{ DIA 04/05 } & \multicolumn{2}{|r|}{ DIA 06/05 } & \multicolumn{2}{|r|}{ DIA 08/05 } & \multicolumn{2}{|r|}{ DIA $10 / 05$} \\
\hline PONTOS & TEMPERATURA ${ }^{\circ} \mathrm{C}$ & PONTOS & TEMPERATURA ${ }^{\circ} \mathrm{C}$ & PONTOS & TEMPERATURA ${ }^{\circ} \mathrm{C}$ & PONTOS & TEMPERATURA ${ }^{\circ} \mathrm{C}$ & PONTOS & TEMPERATURA ${ }^{\circ} \mathrm{C}$ \\
\hline P1 & 23 & P1 & 26 & P1 & 25 & P1 & 23 & P1 & 24 \\
\hline P2 & 23 & P2 & 26,5 & P2 & 25 & P2 & 23 & P2 & 22 \\
\hline P3 & 23 & P3 & 26 & P3 & 25 & P3 & 22 & P3 & 22 \\
\hline P4 & 22,5 & P4 & 26 & P4 & 25 & P4 & 22 & P4 & 22,5 \\
\hline P5 & 22 & P5 & 27 & P5 & 25 & P5 & 22 & P5 & 21 \\
\hline P6 & 22 & P6 & 26,5 & P6 & 26 & P6 & 22 & P6 & 22 \\
\hline P7 & 21 & P7 & 27 & P7 & 25 & P7 & 22 & P7 & 21 \\
\hline P8 & 21 & P8 & 27 & P8 & 26 & P8 & 21 & P8 & 20,5 \\
\hline P9 & 21 & P9 & 26,5 & P9 & 25 & P9 & 21 & P9 & 20,5 \\
\hline P10 & 20 & P10 & 26 & P10 & 26 & P10 & 21 & P10 & 20 \\
\hline P11 & 20 & P11 & 26 & P11 & 25 & P11 & 20,5 & P11 & 20 \\
\hline P12 & 20 & P12 & 26,5 & P12 & 25 & P12 & 21 & P12 & 20,5 \\
\hline P13 & 20 & P13 & 25,5 & P13 & 24 & P13 & 20,5 & P13 & 20,5 \\
\hline P14 & 20 & P14 & 25 & P14 & 25 & P14 & 21 & P14 & 20 \\
\hline P15 & 20 & P15 & 25 & P15 & 26 & P15 & 21 & P15 & 20,5 \\
\hline P16 & 20 & P16 & 25,5 & P16 & 25 & P16 & 21 & P16 & 20,5 \\
\hline P17 & 20 & P17 & 25,5 & P17 & 25 & P17 & 21 & P17 & 20 \\
\hline P18 & 20 & P18 & 25 & P18 & 25 & P18 & 21 & P18 & 20 \\
\hline
\end{tabular}


Os dados levantados pelo grupo foram levados para a sala de aula para serem discutidos e comparados com em outros experimentos similares, o que permitiu chegar em uma conclusão mais consolidada em relação a como o uso do solo pode influenciar diretamente o microclima de uma região.

\section{REFERÊNCIAS BIBLIOGRÁFICAS}

AMORIM, Margarete Cristiane de Costa Trindade. Intensidade e forma da ilha de calor urbana em Presidente Prudente/SP: episódios de inverno. Geosul, Florianópolis, v. 20, n. 39, p 65-82, jan./jun. 2005;

AMORIM, Margarete Cristiane de Costa Trindade. ILHAS DE CALOR EM BIRIGUI/SP. Revista Brasileira de Climatologia, v. 1, n. 1, p 121-130, dez. 2005; 